\title{
Adénocarcinome polymorphe de bas grade : une localisation labiale
}

\author{
Fabrice Campana ${ }^{1, *}$, Nicolas Fakhry ${ }^{2,3}$, Jean Del Grande4, Ugo Ordioni ${ }^{1}$ \\ Centre massilien de la face, Marseille, France \\ Service d'ORL, centre hospitalier universitaire de la Timone, Marseille, France \\ REFCOR (Réseau d'expertise français sur les cancers ORL rares) \\ Service d'anatomopathologie, centre hospitalier universitaire de la Timone, Marseille, France \\ f.campana@centremassiliendelaface.com
}

(Reçu le 20 août 2014, accepté le 31 août 2014)

Mots clés : glandes salivaires mineures / tumeurs des glandes salivaires / adénocarcinome / lèvre
Key words:

salivary glands minor / salivary gland neoplasms / adenocarcinoma / lip
Résumé - Introduction : L'adénocarcinome polymorphe de bas grade (APBG) est une tumeur carcinomateuse épithéliale se développant principalement à partir des glandes salivaires du palais ou des lèvres. Observation : Nous rapportons le cas d'un APBG de la lèvre supérieure. Discussion : La faible spécificité clinique et le polymorphisme histologique de L'APBG rendent son diagnostic difficile. Une réunion de consultation pluridisciplinaire (RCP) du Réseau d'expertise français sur les cancers ORL rares (REFCOR et REFCORpath) a permis le diagnostic histologique et la prise en charge.

\begin{abstract}
Polymorphous low-grade adenocarcinoma: a lip localisation. Introduction: Polymorphous low-grade adenocarcinoma (PLGA) is a carcinomatous epithelial tumour mainly located on the palate or lips. Observation: We report the case of a PLGA of the upper lip. Discussion: The low clinical specificity and histological polymorphism of PLGA make diagnosis difficult. A multidisciplinary consultation meeting of the French network of expertise on rare head and neck cancers (REFCOR and REFCORpath) allowed histological diagnosis and management.
\end{abstract}

\section{Observation}

Un patient de 67 ans s'est présenté à la consultation pour une lésion de la face interne de la lèvre. L'examen endobuccal retrouvait un mauvais état buccal avec des racines dentaires délabrées. Un nodule aplati de $1,5 \mathrm{~cm}$ de diamètre et d'aspect homogène était présent sur la lèvre muqueuse supérieure (Fig. 1). La palpation était souple, les aires ganglionnaires libres.

L'orthopantomographie mettait en évidence une zone d'ostéolyse en regard des 21,22 et 23. La mise en état bucco-dentaire et l'exérèse de la lésion ostéolytique ainsi que du nodule labial ont été réalisées sous anesthésie locale.

L'examen anatomopathologique du nodule muqueux retrouvait une prolifération tumorale riche en cellules globuleuses, à cytoplasme éosinophile, regroupées en amas avec présence de cordons et parfois de tubes à la lumière clarifiée. Il existait de rares mitoses. L'immunohistochimie retrouvait une PS100 positive et un KI67 très légèrement positif (10 à $20 \%$ ). Le diagnostic d'adénocarcinome polymorphe de bas grade (APBG) d'une glande salivaire accessoire était retenu après relecture anatomopathologique effectuée par le réseau REFCORpath. L'histologie de la lésion osseuse était en faveur d'une lésion inflammatoire odontogène.
Le scanner thoraco-abdomino-pelvien et la panendoscopie ORL étaient négatifs. La décision thérapeutique a été prise en RCP nationale dans le cadre du réseau REFCOR. Compte tenu de la faible agressivité (bas grade) et de l'absence d'adénopathie, une reprise des marges d'exérèse (qui n'a pas retrouvé de résidu tumoral) sans geste ganglionnaire a été effectuée. Après 14 mois de surveillance, il n'existait pas de récidive.

\section{Discussion}

L'APBG a été décrit pour la première fois en 1983. Le sexe ratio est de deux femmes pour un homme, le pic d'incidence se situe autour de la sixième décade [1]. Il est, en termes de fréquence, la deuxième tumeur carcinomateuse des glandes salivaires [2,3], avec une localisation quasi élective pour les glandes accessoires. Le site le plus fréquent est le palais dur, la lèvre supérieure ne représentant que moins de $10 \%$ des cas $[3,4]$. Cliniquement, l'APBG se présente comme un nodule jaunâtre dont la taille moyenne est de plus de $2 \mathrm{~cm}$ de diamètre. Le bilan paraclinique tomodensitométrique ou par résonance magnétique est non spécifique et permet d'apprécier l'extension de la tumeur [5]. Il est caractérisé histologiquement par un monomorphisme cellulaire, un polymorphisme architectural 

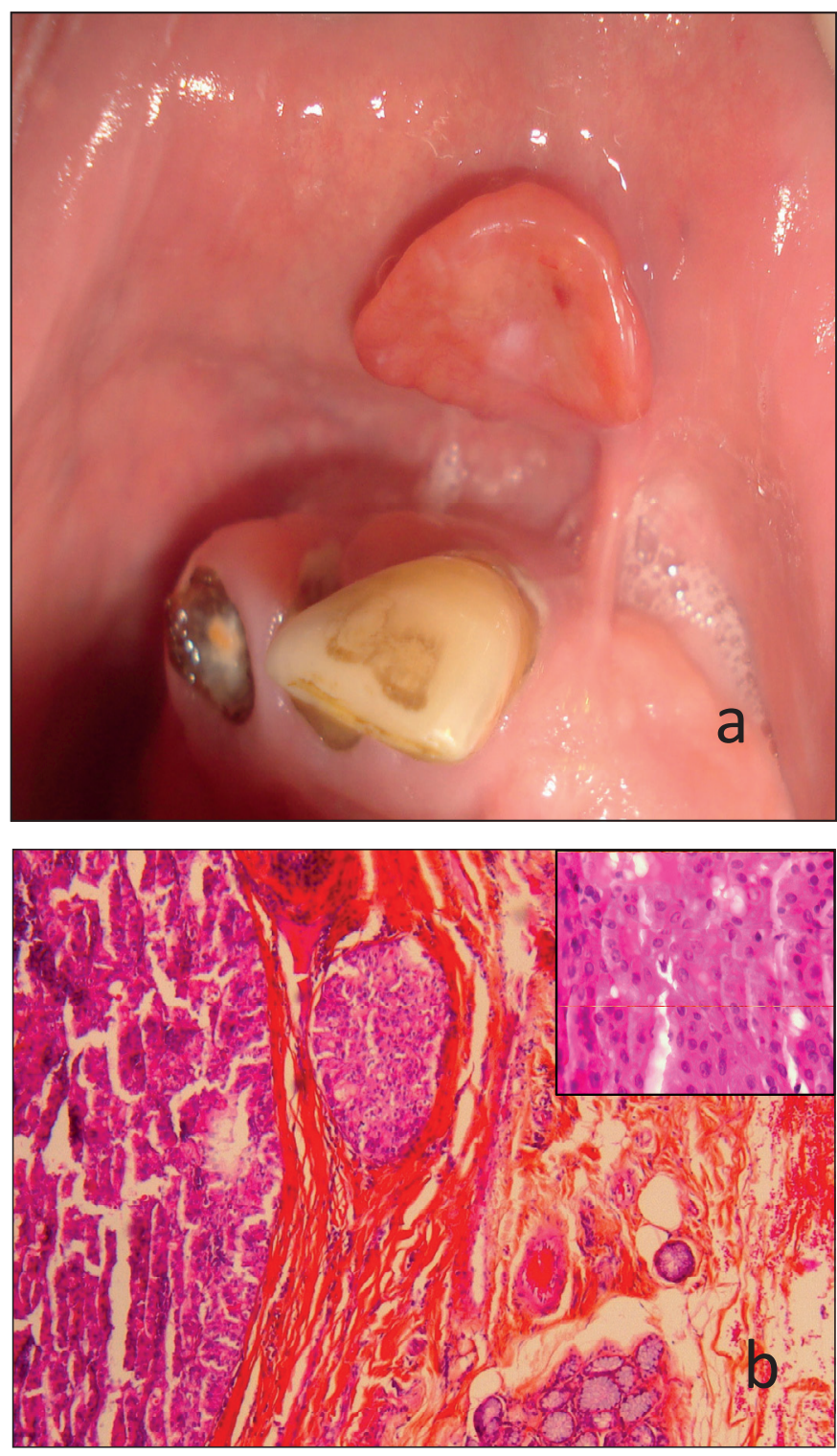

Fig. 1. a : aspect clinique de la lésion labiale : nodule aplati de $1,5 \mathrm{~cm}$ de diamètre et d'aspect homogène. $b$ : coupe histologique de la lésion, coloration HES $\times 100$ : tumeur mal limitée, avec la présence de cellules peu cohésives. À plus fort grossissement (× 400), détails nucléaires évoquant le bas grade de la tumeur.

Fig. 1. a: clinical appearance of lip lesion: flattens nodule, $1.5 \mathrm{~cm}$ in diameter and uniform appearance. $b$ : histological section of the lesion, HES staining $\times 100$ : ill-defined tumor with the presence of low cohesive cells. At higher magnification ( $\times 400)$, nuclear detail evoking the low grade of the tumor.

et un mode de croissance invasif [2]. Les différentes configurations architecturales sont lobulaires, cribiformes, papillaires et trabéculaires [2]. Ce polymorphisme rend le diagnostic histologique difficile $[1,4]$. Dans notre cas, plusieurs lectures des lames n'ont pas permis d'avoir une certitude diagnostique. Une relecture par le réseau REFCORpath a permis de poser le diagnostic d'APBG. Le diagnostic différentiel doit se faire avec un carcinome adénoïde kystique [6] dans sa localisation palatine ou avec un adénome pléomorphe [7].

Le traitement consiste en une exérèse chirurgicale large potentiellement associée à un curage ganglionnaire et à une radiothérapie [7]. La RCP nationale n'a retenu dans notre cas qu'une reprise des marges chirurgicales.

Le pronostic de l'APBG est favorable. Les récidives locales sont possibles dans 10 à $25 \%$ des cas avec une notion de plus grande agressivité [4]. Les métastases sont rares (10\%) [2]. Toutefois l'APBG nécessite une longue période de surveillance, la récidive moyenne apparaissant à 15 ans [1].

\section{Conflits d'intérêt : aucun}

\section{References}

1. Fife TA, Smith B, Sullivan CA, Browne JD, Waltonen JD. Polymorphous low-grade adenocarcinoma: a 17 patient case series. Am J Otolaryngol 2013;34:445-448.

2. Thompson L. World Health Organization classification of tumours: pathology and genetics of head and neck tumours. Ear Nose Throat J 2006;85:74.

3. Buchner A, Merrell PW, Carpenter WM. Relative frequency of intra-oral minor salivary gland tumors: a study of 380 cases from northern California and comparison to reports from other parts of the world. J Oral Pathol Med 2007;36:207-214.

4. Sankar Vinod V, Mani V, George A, Sivaprasad KK. Polymorphous low-grade adenocarcinoma-management and reconstruction with temporalis myofacial flap. J Maxillofac Oral Surg 2013;12:105-108.

5. Kato H, Kanematsu M, Makita H, Kato K, Hatakeyama D, Shibata T, et al. CT and MR imaging findings of palatal tumors. Eur J Radiol 2014;83:e137-146.

6. El-Nagdy S, Salama NM, Mourad MI. Immunohistochemical clue for the histological overlap of salivary adenoid cystic carcinoma and polymorphous low-grade adenocarcinoma. Interv Med Appl Sci 2013;5:131-139.

7. Chaâbouni S, Ayadi L, Dhouib H, Abbes K, Khabir A, Boudawara T. Polymorphous low-grade adenocarcinoma: a palatine and a labial location. Rev Stomatol Chir Maxillofac 2008;109:178-182. 